\title{
Management Function Deployment: um Método para o Alinhamento Estratégico da Melhoria dos Processos de Gerenciamento de Projetos de Software
}

\author{
Gustavo H. P. Carvalho, Fabio Q. B. da Silva, A. César C. França \\ Centro de Informática - Universidade Federal de Pernambuco (UFPE) \\ Caixa Postal 7851 - 50.732-970 - Recife - PE - Brasil \\ \{ghpc, fabio\}@cin.ufpe.br, cesarfranca@gmail.com
}

\begin{abstract}
Resumo. Este artigo apresenta um método, denominado Management Function Deployment (MFD), que permite alinhar a melhoria dos processos de gerenciamento de projetos de software aos objetivos estratégicos organizacionais. Este alinhamento é importante para aumentar a efetividade da melhoria de processos, mas ainda são poucos os guias práticos de como realizá-lo. Para avaliar a aplicabilidade industrial deste método, um estudo de campo foi realizado em uma empresa de desenvolvimento de software. Como resultado, os participantes avaliaram positivamente o uso do MFD. Por fim, analisa-se a generalização do resultado para outros contextos.
\end{abstract}

\begin{abstract}
This article presents a method, named Management Function Deployment (MFD), which allows alignment of project management process improvement with organizational level strategies. The relevance of this alignment is to increase the effectiveness of the process improvement provided that there are not many practical guidelines on how to achieve it. To evaluate the industrial applicability of this method, a field study was carried out on a software development company. The participants from these companies approved the MFD. Further and general MFD applications are discussed for other contexts.
\end{abstract}

\section{Introdução}

Aumentar a maturidade dos processos de gerenciamento dos projetos é uma preocupação materializada pelos vários modelos de maturidade em gerenciamento de projetos existentes. Porém, deseja-se também aumentar a importância estratégica da melhoria de processos. Este desejo baseia-se na premissa de que alinhar as práticas de gerenciamento de projetos às estratégias do negócio facilita significativamente o alcance de metas e estratégias organizacionais [SRIVANNABOON, 2006].

No entanto, apesar de alguns modelos de maturidade em gerenciamento de projetos já terem incorporado a preocupação com o alinhamento estratégico, ainda são poucos os estudos que oferecem guias práticos de como alcançar este alinhamento. Portanto, é nesta lacuna em que se baseia o problema de pesquisa deste trabalho: como alinhar a melhoria dos processos de gerenciamento de projetos de software aos objetivos estratégicos de uma organização, de forma que maior atenção seja conferida aos processos de gerenciamento mais relacionados à estratégia da empresa.

Para tanto, desenvolveu-se um método, denominado Management Function Deployment (MFD), o qual se baseia no método Quality Function Deployment(QFD) 
(COHEN, 1995). Desta forma, através do MFD, é possível identificar, quais processos de gerenciamento de projetos devem ser priorizados em função dos objetivos estratégicos traçados pela organização.

Para avaliar a adequação e a viabilidade do uso prático do MFD, foi realizado um estudo de campo no âmbito de uma empresa de desenvolvimento de software localizada em Recife, Pernambuco. A partir das análises realizadas, nos dados coletados no estudo de campo, foram identificados os principais aspectos positivos e oportunidades de melhoria do MFD. Portanto, as principais contribuições deste trabalho são: o método Management Function Deployment, exemplos práticos de sua utilização, além de uma análise crítica sobre o mesmo.

$\mathrm{Na}$ Seção 2 é apresentado o referencial teórico da pesquisa e os principais trabalhados relacionados. Na Seção 3 é descrito o método de pesquisa. Na Seção 4 o método MFD é descrito e, em paralelo, apresenta-se um exemplo de uso retirado do estudo de campo realizado. Na seção 5 faz-se uma análise crítica do MFD a partir das informações levantadas no estudo de campo. Por fim, a seção 6 traz conclusões e recomendações de trabalhos futuros.

\section{Trabalhos Relacionados}

Desde que se iniciou o estudo do gerenciamento de projetos em organizações, existe uma importante preocupação em achar um bom alinhamento entre o gerenciamento de projetos e as estratégias organizacionais [YUMING; QUAN; GUO, 2007].

Este alinhamento tem sido estudado sobre várias perspectivas. Harrison e Lock (2004) utilizam áreas funcionais (por exemplo, produção e recursos humanos) como variáveis para examinar o alinhamento entre as práticas de gerenciamento de projetos e as estratégias funcionais. Já Englund e Graham (1999) estudam o alinhamento às estratégias de negócio através de políticas de seleção de projetos. Os estudos de Milosevic e Srivannaboon (2006) especializam a abordagem de Englund e Graham a partir da identificação de atributos competitivos das estratégias do negócio, que irão influenciar a seleção dos projetos. Este artigo segue uma linha distinta destas antes mencionadas. O foco deste trabalho é priorizar a melhoria dos processos de gerenciamento de projetos que mais impactam positivamente as metas estratégicas traçadas pela organização.

Carnevalli e Miguel (2008) mostram que alguns trabalhos têm usado o método Quality Function Deployment (QFD) para desenvolver as estratégias organizacionais através do alinhamento da melhoria de processos a estas. Porém, estes trabalhos não têm foco específico na melhoria dos processos de gerenciamento de projetos.

O QFD foi originalmente desenvolvido no Japão em 1966 por Yoji Akao que combinou seu trabalho sobre garantia e controle da qualidade com a técnica de desdobramento de funções usada pela engenharia. De acordo com Cohen (1995), QFD é uma técnica para o planejamento e desenvolvimento estruturado de produtos que permite especificar, de forma clara, as necessidades dos clientes e, então, analisar sistematicamente o impacto dos produtos propostos na satisfação destas necessidades.

A House of Quality (HoQ) constitui o principal instrumento do QFD. Esta é um conjunto matrizes relacionadas entre si. A HoQ é formada por seis matrizes (Figura 1) que possuem, respectivamente, as seguintes informações: (1) as necessidades dos clientes, (2) como estas necessidades são satisfeitas atualmente pela empresa e pelos concorrentes, (3) as soluções propostas pela empresa para atender estas necessidades, 
(4) como estas soluções satisfazem positivamente ou negativamente as necessidades dos clientes, (5) as dependências existentes entre as soluções propostas e (6) a priorização das soluções que mais contribuem para a satisfação das necessidades dos clientes.

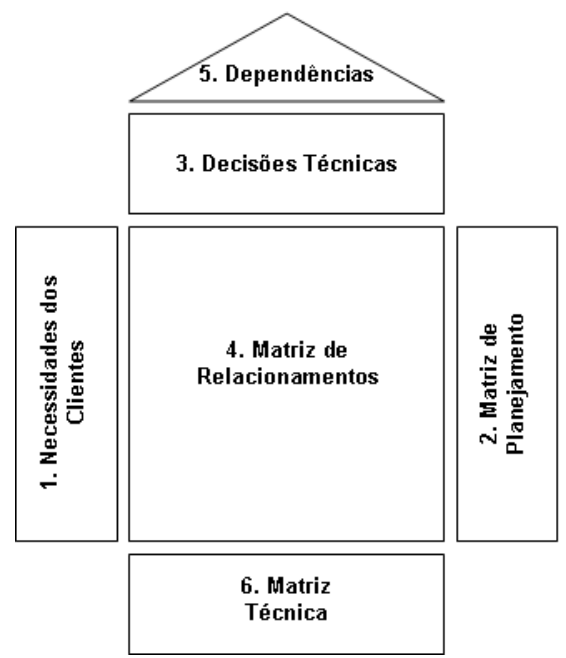

Figura 1 - As Matrizes da House of Quality (HoQ).

Recentemente, algumas adaptações têm sido propostas para o QFD com o objetivo de analisar sistematicamente o impacto da melhoria de processos às estratégias organizacionais. Zhao et al. (2003) utilizam o QFD como instrumento para a reengenharia de processos de negócio em função das estratégias organizacionais. DongSheng e Feng-Xia (2007) propõem também um modelo de otimização dos processos de negócios organizacionais baseado no QFD.

Mais alinhados a este trabalho têm-se os estudos de Liu et al. (2005) e Becker, Prikladnicki e Audy (2008) que adaptam o QFD para priorizar a melhoria dos processos do CMM-SW e do CMMI-DEV, respectivamente, em função das metas estratégicas traçadas. Contudo, o trabalho aqui desenvolvido difere destes dois pelas seguintes características:

1. A priorização considera a dificuldade de sair do nível atual para o nível desejado de satisfação da meta estratégica.

2. A priorização considera a dificuldade de sair do nível atual para o nível desejado de capacidade dos processos de gerenciamento de projetos.

3. A priorização considera, além do impacto neutro e positivo, impactos negativos da melhoria dos processos nas metas estratégicas.

4. Por fim, a adaptação aqui proposta tem foco específico na melhoria de processos de gerenciamento de projetos a partir da visão dos modelos de maturidade em gerenciamento de projetos.

Estudos recentes mostram que o interesse pelos modelos de maturidade em gerenciamento de projetos é elevado: de 373 empresas brasileiras pesquisadas, $19 \%$ conhecem e utilizam estes modelos, $32 \%$ conhecem e pretendem utilizar e $37 \%$ não conhecem, mas consideram a idéia interessante [PINTO, 2008]. Atualmente, os modelos de maturidade em gerenciamento de projetos mais referenciados são: o Organizational Project Management Maturity Model (OPM3) [PMI, 2008]; o Project Management Maturity Model (PMMM) [CRAWFORD, 2006]; o Portfolio, Programme 
and Project Management Maturity Model (P3M3) [OGC, 2008]; o PRINCE2 Maturity Model (P2MM) [OGC, 2006]; o Kerzner Project Management Maturity Model (KPMMM) [KERZNER, 2005] e o Modelo de Maturidade em Gerenciamento de Projetos (MMGP) [PRADO, 2008]. Carvalho, Vasconcelos e Da Silva (2009) estabelecem 18 critérios e a partir destes comparam em detalhes cada um destes modelos.

\section{Método de Pesquisa}

Esta pesquisa foi estruturada em uma sequiência de cinco fases, seguindo as orientações de Easterbrook e Aranda (2009), descritas a seguir.

1. Fase I - Levantamento do Conhecimento Prévio: a primeira fase teve como objetivo estudar e compreender o conhecimento prévio, assim como teorias existentes e trabalhos relacionados com esta pesquisa. Este estudo inicial da área considerou materiais selecionados de maneira ad hoc.

2. Fase II - Estudo das Soluções Existentes: na segunda fase, uma análise estruturada dos principais trabalhos diretamente relacionados ao problema de pesquisa foi realizada. O objetivo foi encontrar as limitações dos estudos em relação ao alinhamento estratégico da melhoria de processos.

3. Fase III - Elaboração da Solução Proposta: a terceira fase teve como objetivo elaborar a solução proposta por este trabalho; no caso, um método baseado no QFD para o alinhamento estratégico da melhoria dos processos de gerenciamento de projetos.

4. Fase IV - Desenho do Estudo de Campo: na quarta fase foi planejado o estudo de campo com o objetivo de avaliar pontos fortes e fracos em relação à aplicabilidade do MFD no contexto industrial. Nesta fase, foram selecionados, por conveniência, os sujeitos do estudo de campo entre as empresas de desenvolvimento de software de Recife, PE.

5. Fase V - Realização do Estudo de Campo: na quinta fase o estudo de campo foi realizado e os resultados foram analisados produzindo refinamentos no método e confirmando sua aplicabilidade prática. Utilizou-se uma abordagem qualitativa, através de entrevistas semi-estruturadas em profundidade.

\subsection{Caracterização do Estudo de Campo}

O estudo de campo foi realizado em janeiro de 2010 em uma empresa de desenvolvimento de software fundada em 2006. Pela classificação de empresas do SEBRAE, é uma pequena empresa (10 a 49 funcionários) que teve uma receita bruta em 2009 entre $\mathrm{R} \$ 1.000 .000$ e $\mathrm{R} \$ 2.400 .000$.

Para a coleta de dados foi realizada uma entrevista semi-estruturada simultânea com dois representantes da organização envolvidos na sua gestão estratégica. $\mathrm{O}$ primeiro (Participante A) é diretor comercial e gerente de produtos e projetos, enquanto que o segundo (Participante B) é diretor administrativo e financeiro da empresa. Ambos estão há 4 anos na organização e julgaram como médio o nível de seus conhecimentos em gerenciamento de projetos.

Este estudo teve uma duração total de 2 horas e 46 minutos para analisar o alinhamento de 2 das 6 estratégias organizacionais. Os 9 minutos iniciais foram utilizados para apresentar os objetivos da pesquisa, fazer uma breve descrição do MFD 
e levantar alguns dados pessoais sobre os participantes. O tempo restante foi alocado para cada fase do ciclo do MFD, descrito na Seção 4.

\subsection{Limitações da Pesquisa}

Esta pesquisa possui como principal limitação a avaliação do método proposto em um único estudo de campo selecionado por conveniência. Esta limitação deve-se à dificuldade de encontrar empresas dispostas a participar do estudo, que requer a aplicação de questionários em entrevistas de longa duração (em torno de três horas). Para minimizar esta limitação, procurou-se caracterizar bem o uso do MFD com o intuito de permitir a repetição do estudo de campo realizado em outros contextos organizacionais, semelhantes ou diferentes.

\section{Management Function Deployment (MFD)}

O método Management Function Deployment segue, em linhas gerais, a filosofia do método Quality Function Deployment (QFD). O QFD é um método para o planejamento estruturado de novos produtos, que avalia cada produto proposto em termos da contribuição deste na satisfação das necessidades dos clientes. De forma similar, MFD é um método para o planejamento estruturado da melhoria dos processos de gerenciamento de projetos, que avalia cada melhoria em termos da contribuição desta na satisfação dos objetivos estratégicos da organização. Nesta seção, os conceitos do MFD são apresentados e ilustrados com exemplos de sua aplicação a partir dos dados extraídos do estudo de campo, caracterizado na Seção 3.1.

O MFD é composto por dois instrumentos e um processo cíclico que guia e estrutura a criação destes instrumentos. Estes instrumentos são dois conjuntos de matrizes (Figura 2): a House of Management (HoM) e a House of Actions (HoA).

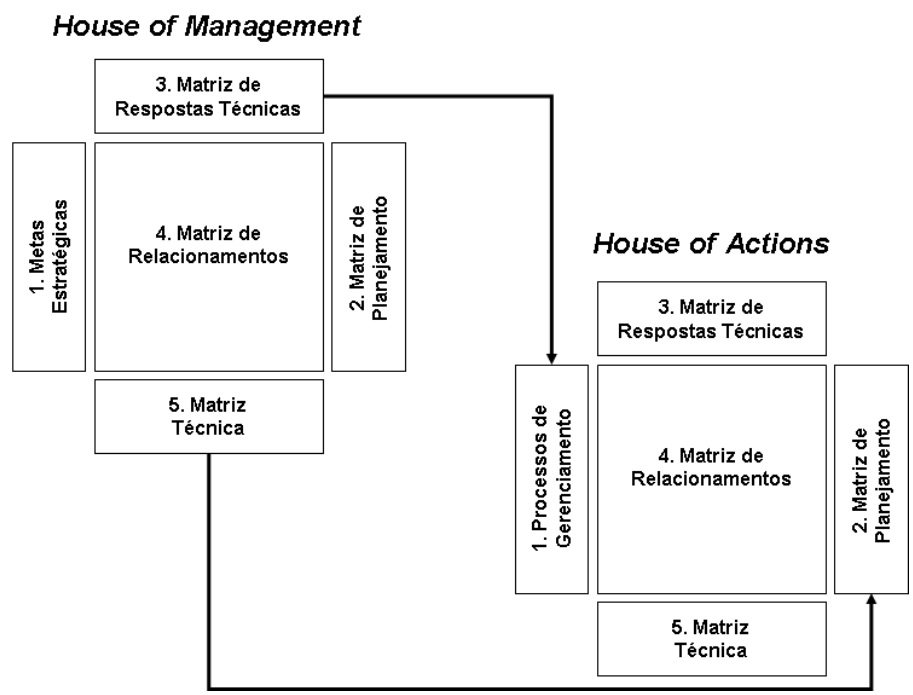

Figura 2 - Os Instrumentos HoM e HoA do MFD.

A HoM é composta por um conjunto de cinco matrizes (adaptadas a partir da House of Quality (HoQ) do QFD) que permite alinhar a melhoria dos processos de gerenciamento de projetos às estratégias organizacionais:

- Matriz de Metas Estratégicas: contém as metas estratégicas da organização, construídas em algum processo de planejamento estratégico (externo ao MFD). 
- Matriz de Planejamento: contém informações sobre a situação atual e desejada da organização em relação às suas metas estratégicas.

- Matriz de Respostas Técnicas: contém a lista dos processos de gerenciamento de projetos considerados pela organização (aderentes a algum modelo ou guia de processos ou definidos de forma ad hoc pela organização).

- Matriz de Relacionamentos: contém o nível de influência positiva, negativa ou neutra da melhoria dos processos de gerenciamento de projetos em relação às metas estratégicas organizadas na Matriz de Metas Estratégicas.

- Matriz Técnica: contém a capacidade atual e projetada dos processos de gerenciamento de projetos. A partir destas e outras informações, prioriza-se a melhoria dos processos de gerenciamento de projetos, com o intuito de maximizar o nível de influência positiva desta em relação às metas estratégicas.

A HoA é composta por um conjunto de cinco matrizes derivadas da HoM que permite alinhar ações de melhoria aos processos de gerenciamento de projetos priorizados pela HoM. Portanto, a HoA tem como entrada dados da HoM, conforme ilustrado na Figura 2. As matrizes da HoA são descritas a seguir:

- Matriz de Processos de Gerenciamento: contém os processos de gerenciamento de projetos presentes na Matriz de Respostas Técnicas da HoM.

- Matriz de Planejamento: contém informações sobre a priorização da melhoria dos processos de gerenciamento de projetos feita na Matriz Técnica da HoM.

- Matriz de Respostas Técnicas: contém ações de melhoria relacionadas com os processos listados na primeira matriz da HoA.

- Matriz de Relacionamentos: contém o nível de influência positiva, negativa ou neutra das ações de melhoria em relação aos processos de gerenciamento de projetos organizados na primeira matriz da HoA.

- Matriz Técnica: contém a priorização das ações de melhoria a partir das demais informações da HoA. O objetivo é priorizar as ações com maior influência positiva sobre os processos mais prioritários.

O processo cíclico que guia e estrutura a construção da HoM e da HoA é formado por oito etapas, ilustradas na Figura 3. Este processo padroniza como as matrizes do MFD serão preenchidas e cria um ambiente para se estabelecer um ciclo de melhoria contínua dos processos de gerenciamento de projetos alinhada às estratégias organizacionais. As subseções a seguir descrevem as atividades em cada etapa deste ciclo e ilustram a construção das matrizes com exemplos extraídos do estudo de campo. 


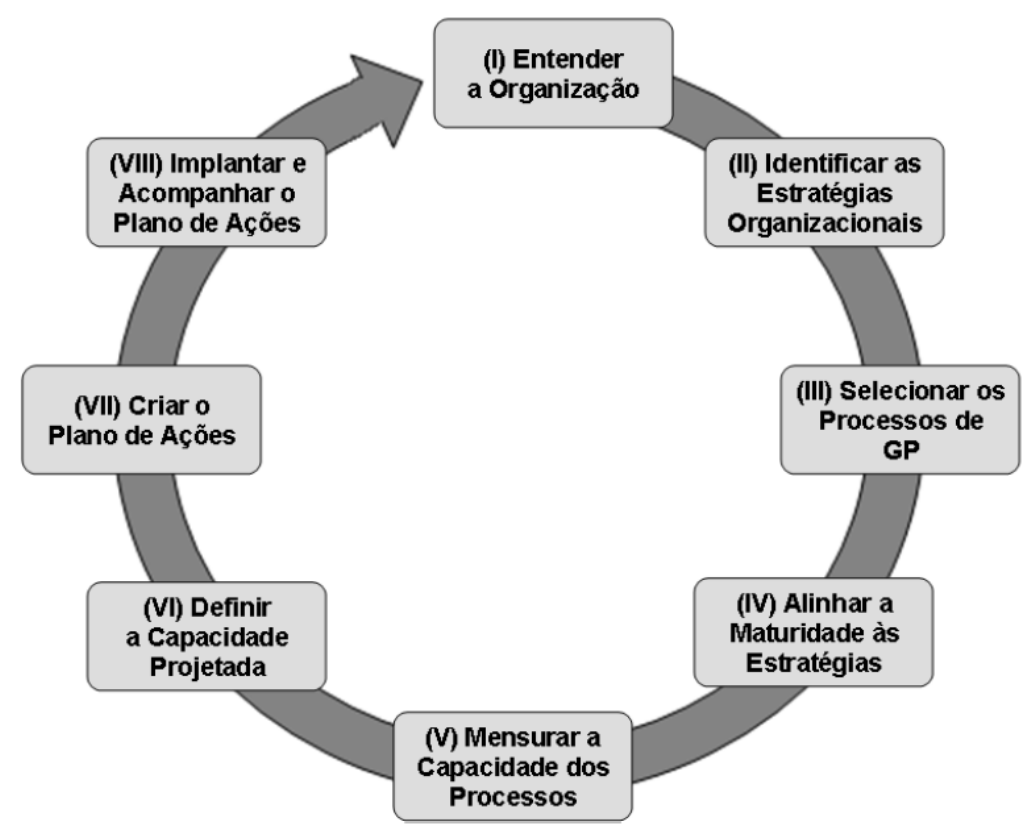

Figura 3 - Etapas Prescritas pelo MFD para a Construção da HoM e HoA.

\subsection{Etapa I - Entender a Organização}

O objetivo desta etapa é levantar e documentar aspectos organizacionais que devem ser levados em consideração tanto para caracterizar a organização envolvida quanto para refletir sobre a realidade da empresa. Este levantamento permite analisar eventuais desdobramentos que a realidade de cada empresa pode ter sobre a aplicação do MFD. Para tanto, faz-se uso de um questionário para compreensão preliminar da organização. que é composto pelas seguintes seções:

1. Dados gerais da organização.

2. Dados mercadológicos.

a. Para a organização participante do estudo de campo: esta tem como principais mercados a consultoria e o desenvolvimento de software, nos quais atua há quatro anos. De acordo com os participantes, seus principais pontos fortes são: qualidade técnica, baixo custo e agilidade em mudanças. Já os pontos a melhorar são: comercial (vendas), divulgação (reconhecimento no mercado) e rede de relacionamentos.

3. Dados da maturidade organizacional e certificações de qualidade.

a. Para a organização participante do estudo de campo: não possui nenhuma certificação de qualidade de processos ou produtos.

4. Dados do planejamento estratégico.

a. Para a organização participante do estudo de campo: realiza sistematicamente planejamento estratégico. O primeiro foi realizado em 2008 e o último, ainda não concluído, em 2010. Para tanto, utiliza planejamento de cenários, análise SWOT e diagrama de Ishikawa.

5. Dados da maturidade em gerenciamento de projetos. 
a. Para a organização participante do estudo de campo: esta não possui o papel de gerente de projetos bem definido. Porém, as práticas de gerenciamento seguem parte das recomendações do PMBOK e do Scrum. Por fim, não existe nenhuma política de melhoria contínua dos processos de gerenciamento de projetos.

A Etapa I precisou de 16 minutos para ser realizada.

\subsection{Etapa II - Identificar as Estratégias Organizacionais}

O objetivo desta etapa é levantar e documentar as estratégias organizacionais. As estratégias são um dos principais insumos para a construção do MFD, pois a melhoria dos processos deverá ser alinhada a estas. Assume-se aqui que a empresa deve ter realizado previamente um planejamento estratégico, uma vez que o MFD não contempla a realização deste. As metas estratégicas traçadas pela organização devem ser documentadas na matriz de Metas Estratégicas da HoM.

No MFD a Matriz de Planejamento prioriza as metas estratégicas presentes na Matriz de Metas Estratégicas. A Matriz de Planejamento é composta pelas colunas:

1. Importância: mede a importância da respectiva meta estratégica para a organização quando comparada com as demais metas. Utiliza uma escala Likert na qual 1 significa menor importância e 5 maior importância.

2. Satisfação atual: indica o quão perto ou longe a organização está de atingir a meta estratégica estabelecida. Também de acordo com uma escala Likert na qual 1 significa mais longe e 5 significa que a meta já foi alcançada.

3. Satisfação desejada: indica o quanto a organização deseja caminhar em direção à meta. Utiliza a mesma escala da satisfação atual.

4. Taxa de melhoria: representa a dificuldade de sair da satisfação atual e alcançar a satisfação desejada para a respectiva meta estratégica. Assim como o QFD, o MFD assume três possibilidades de cálculo.

a. A divisão da satisfação desejada pela satisfação atual, para os casos onde as melhorias iniciais são mais difíceis do que melhorias posteriores.

b. A subtração da satisfação desejada pela satisfação atual mais um, para os casos onde a dificuldade de melhoria é linear; ou seja, sair do nível 1 para o 2 é tão difícil quanto sair do 2 para o 3 .

c. Uma escala específica que denota a dificuldade pelo ponto de vista do respondente: 1, para os casos onde a satisfação desejada já é a atual; 1.2 para as melhorias de dificuldade moderada e 1.5 para as melhorias difíceis.

Por padrão, o MFD assume a segunda fórmula de cálculo. Contudo, caso esta fórmula não reflita o entendimento da organização sobre a dificuldade da melhoria, uma das outras fórmulas deve ser utilizada.

5. Peso absoluto: combina a importância da meta para a organização com a taxa de melhoria multiplicando estes valores. Assim como o QFD, o MFD priorizará as melhorias mais difíceis e com maior importância para o respondente. Ou seja, quanto maior o peso absoluto, maior é a prioridade da respectiva meta.

6. Peso normalizado: artifício matemático para normalizar o peso absoluto. 
7. Peso normalizado acumulado: representa o acúmulo do peso normalizado de várias metas estratégicas.

A Figura 4 mostra a Matriz de Metas Estratégicas e a Matriz de Planejamento preenchidas de acordo com os dados levantados no estudo de campo.

\begin{tabular}{|l|l|c|c|c|c|c|c|c|}
\hline & Importância & Satisfação Atual & Satisfação Desejada & Taxa de Melhoria & Peso Absoluto (PA) & PA Normalizado (PAN) & PAN Acumulado \\
\hline Ter processos internos claros para sócios e colaboradores & 3 & 3 & 5 & 3,00 & 9,00 & 0,16 & 0,16 \\
\hline Expansão da rede de contatos (networking) & 4 & 3 & 4 & 2,00 & 8,00 & 0,14 & 0,30 \\
\hline Aumento (dobrar) o faturamento/lucro & 4 & 2 & 4 & 3,00 & 12,00 & 0,21 & 0,51 \\
\hline Redução da carga horária administrativa dos sócios & 4 & 4 & 5 & 2,00 & 8,00 & 0,14 & 0,65 \\
\hline Gerar produtos próprios & 5 & 3 & 5 & 3,00 & 15,00 & 0,26 & 0,91 \\
\hline Obter financiamento para projetos & 5 & 5 & 5 & 1,00 & 5,00 & 0,09 & 1,00 \\
\hline
\end{tabular}

Figura 4 - Matriz de Metas Estratégicas e de Planejamento (HoM).

No estudo de campo, as etapas II e IV foram realizadas em conjunto, consumindo 72 minutos.

\subsection{Etapa III - Selecionar os Processos de GP}

Na terceira etapa do MFD, deve-se preencher a Matriz de Respostas Técnicas com a lista dos processos de gerenciamento de projetos que serão considerados na priorização. A organização é livre para escolher estes processos a partir da fonte que considerar mais adequada ou, inclusive, escolher estes a partir do que a organização considera como bons processos de gerenciamento de projetos. No entanto, aconselha-se que seja utilizado um modelo de maturidade em gerenciamento projetos, uma vez que em etapas futuras será preciso avaliar o nível de capacidade dos processos informados nesta etapa. Caso estes sejam escolhidos a partir de uma fonte que não tenha associado um modelo de avaliação, a organização terá também que elaborar este instrumento.

Desta forma, por padrão, o MFD aconselha que um dos modelos de maturidade em gerenciamento de projetos listados no fim da seção 2 seja selecionado. A priori, a organização deve estudar cada um destes modelos e então escolher aquele que melhor se enquadra na sua realidade. Como esta atividade pode consumir um tempo significativo, o MFD sugere utilizar o processo de recomendação de modelos de maturidade em gerenciamento de projetos descrito por Carvalho, Vasconcelos e Silva (2009) que indica quais modelos são mais adequados a um dado contexto de avaliação, conferindo uma maior pontuação àqueles mais recomendados.

No estudo de campo, utilizou-se como fonte dos processos de gerenciamento de projetos o Project Management Maturity Model (PMMM) [CRAWFORD, 2006]. Esta escolha é embasada por duas razões: primeiro que este modelo de maturidade em gerenciamento tem sua estrutura baseada diretamente no PMBOK 2004, o que facilita a sua compreensão, uma vez que trata de processos bem conhecidos por uma quantidade significativa de gerentes. Segundo que, entre o OPM3 e o PMMM, ambos de alguma forma alinhados ao PMBOK, o PMMM é o que possui menor custo de aquisição, inclusive do respectivo modelo de avaliação. Ou seja, desta forma foi possível do ponto de vista financeiro, dentro do escopo desta pesquisa, aplicar o MFD em casos práticos.

\subsection{Etapa IV - Alinhar a Maturidade às Estratégias}

O objetivo desta etapa é identificar relacionamentos de influência positiva, negativa ou neutra existentes entre a melhoria dos processos de gerenciamento escolhidos na Etapa III e as metas estratégicas levantadas na Etapa II. 
O impacto reflete a influência negativa, neutra ou positiva da melhoria de um dado processo de gerenciamento de projetos em relação a uma dada meta estratégica. Em outras palavras, se a capacidade deste processo for melhorada o que acontece em relação a uma dada meta estratégica: diminuem-se as chances de alcançá-la (impacto negativo), aumentam-se as chances de alcançá-la (impacto positivo) ou as chances permanecem as mesmas (impacto neutro). O impacto é medido conforme uma escala Likert $(-9,-3,-1,0,1,3,9)$ na qual os extremos indicam um alto impacto negativo e positivo, respectivamente. 0 simboliza a ausência de impacto (impacto neutro).

O valor do impacto é atribuído a partir do julgamento consensual daqueles que estão preenchendo as matrizes do MFD. Este valor deve ser registrado na Matriz de Relacionamento para cada linha e coluna desta matriz. Desta forma, cada valor irá representar o impacto da melhoria do $j$-ésimo processo em relação a $i$-ésima meta.

A partir do impacto, é possível calcular o nível de relacionamento existente entre a melhoria de um processo de gerenciamento de projetos e uma meta estratégica. De acordo com o QFD, o relacionamento é a multiplicação do impacto pelo respectivo peso absoluto normalizado. Logo, de forma análoga, o MFD utiliza a mesma fórmula de cálculo. Este valor computado não é explicitamente armazenado em nenhuma matriz, mas é utilizado para o cálculo da contribuição algébrica e absoluta, como será explicado a seguir. $\mathrm{O}$ valor da contribuição é armazenado na Matriz Técnica.

A contribuição de um dado processo em relação às metas estratégicas representa o quanto a melhoria deste processo contribui (positiva ou negativamente) para o alcance das metas. Quanto maior a contribuição, mais a melhoria deste processo ajuda a organização a caminhar na direção de alcançar as suas metas. É a partir deste cálculo que é feito o alinhamento da melhoria dos processos de gerenciamento de projetos em relação às metas estratégicas traçadas. As melhorias com maior contribuição devem ser priorizadas, visto que contribuem mais para o sucesso das metas.

Assim como no QFD, o MFD considera duas contribuições: a algébrica e a absoluta. A contribuição algébrica é o simples somatório do relacionamento da melhoria do processo em questão para todas as metas. Já a contribuição absoluta, não considera o sinal do relacionamento. Ou seja, considera o valor do módulo do relacionamento.

A contribuição absoluta é calculada para que uma importante análise seja feita. Deve-se comparar o valor da contribuição algébrica em relação ao valor da contribuição absoluta. Se o primeiro for muito menor do que o segundo, a contribuição do processo em questão deve ser vista com cuidado.

Se, para um dado processo, a contribuição absoluta é significativamente maior do que a contribuição algébrica, isto indica que possivelmente a melhoria do processo em questão possui um impacto positivo alto para parte das metas, mas também um impacto negativo alto para outra parte das metas. Desta forma, priorizar a melhoria de processos deste tipo fará a organização, ao mesmo tempo, caminhar na direção de algumas metas e se afastar de outras. Para estes casos, aconselha-se dividir o processo em subprocessos, se possível, isolando a parte que traz impactos positivos da que traz impactos negativos. Assim, a organização poderá priorizar não a melhoria do processo como um todo, mas somente o subprocesso no qual predomina os impactos positivos.

Assim como o QFD, o MFD recomenda que se remova da Matriz de Respostas Técnicas as colunas que produzem contribuição algébrica negativa, pois estas representam itens que, de forma global, não trazem benefícios. Além de que valores 
negativos invalidam os cálculos de outros conjuntos de matrizes. A Figura 5 mostra parte da Matriz de Respostas Técnicas, da Matriz de Relacionamentos e da Matriz Técnica preenchida a partir dos dados coletados no estudo de campo.

Por restrições de tempo dos representantes da organização, analisou-se o impacto da melhoria dos 43 processos de gerenciamento de projetos descritos pelo PMMM sobre as metas estratégicas de maior peso absoluto: M3 - Aumentar (dobrar) $o$ faturamento/lucro e M5 - Gerar produtos próprios, cujos pesos absolutos são 0,21 e 0,26, respectivamente. Por isso, a Figura 5 não mostra o impacto para as demais metas.

Dos 43 processos, três se destacaram com maior contribuição. Estes foram: $P 1-$ Execução do PGP, P2 - Aquisição da Equipe e P3 - Desenvolvimento e Gerenciamento do Time do Projeto, todos com contribuição algébrica (e absoluta) igual a 4,26.

\begin{tabular}{|c|c|c|c|c|c|c|c|c|}
\hline & & \multicolumn{7}{|c|}{ Matriz de Respostas Técnicas (Fonte: Project Management Maturity Model) } \\
\hline & & & cursos Humanos & & & Comunic & caçäo & \\
\hline \multirow{2}{*}{\multicolumn{2}{|c|}{ Metas Estratégicas }} & Aquisição da Equipe & \begin{tabular}{|c|} 
Desenvolvimento e \\
Gerenciamento do \\
Time do Projeto
\end{tabular} & $\begin{array}{c}\text { Desenvolvimento } \\
\text { Profissional }\end{array}$ & $\begin{array}{c}\text { Planejamento das } \\
\text { Comunicaçöes }\end{array}$ & $\begin{array}{c}\text { Distribuiçãa das } \\
\text { Informaçōes }\end{array}$ & $\begin{array}{c}\text { Relato do } \\
\text { Desempenho }\end{array}$ & $\begin{array}{l}\text { Gerenciamento e } \\
\text { Rastreamento dos } \\
\text { Problemas }\end{array}$ \\
\hline & & IMPACTO & IMPACTO & IMPACTO & IMPACTO & IMPACTO & IMPACTO & IMPACTO \\
\hline \multicolumn{9}{|c|}{ Ter processos internos claros para sócios e colaboradores } \\
\hline \multicolumn{9}{|c|}{ Expansão da rede de contatos (networking) } \\
\hline \multirow{2}{*}{\multicolumn{2}{|c|}{ Aumento (dobrar) o faturamento/lucro }} & 9 & 9 & 3 & 1 & 3 & 3 & 3 \\
\hline \multicolumn{8}{|c|}{ Redução da carga horária administrtativa dos sócios } & \\
\hline \multirow{2}{*}{\multicolumn{9}{|c|}{\begin{tabular}{|l|} 
Gerar produtos próprios \\
Obter financiamento para projetos
\end{tabular}}} \\
\hline & & & & & & & & \\
\hline \multirow{4}{*}{ Matriz Técnica } & Contribuição Absoluta: & 4,26 & 4,26 & 1,42 & 0,47 & 1,42 & 1,42 & 1,42 \\
\hline & Contribuiçăo Algébrica: & 4,26 & 4,26 & 1,42 & 0,47 & 1,42 & 1,42 & 1,42 \\
\hline & Capacidade Atual: & 2,00 & 1,00 & & & & & \\
\hline & Capacidade Desejada: & 4,00 & 3,00 & & & & & \\
\hline
\end{tabular}

Figura 5 - Matriz de Respostas Ttécnicas, de Relacionamentos e Técnica (HoM).

\subsection{Etapa V - Mensurar a Capacidade dos Processos}

O objetivo desta etapa é mensurar a capacidade atual dos processos de gerenciamento considerados. O resultado da avaliação é devidamente registrado na penúltima linha da Matriz Técnica. Caso esteja-se utilizado um modelo de maturidade em gerenciamento de projetos, deve-se fazer uso do modelo de avaliação deste.

Como toda avaliação de maturidade, esta requer um esforço significativo e demanda um tempo não muito curto. $\mathrm{O}$ mesmo acontecerá aqui. No entanto, caso a organização deseje diminuir o tempo gasto com a avaliação, esta pode avaliar a capacidade somente dos processos com maior contribuição. Desta forma, tem-se uma avaliação parcial, porém mais breve.

Assim procedeu-se no estudo de campo. Os três processos de maior contribuição foram avaliados conforme o PMMM e obtiveram os seguintes valores para capacidade atual (como mostra parcialmente a Figura 5): P1, nível 1; P2, nível 2; P3, nível 1.

\subsection{Etapa VI - Definir a Capacidade Projetada}

O objetivo desta etapa é definir o quanto se deseja melhorar a capacidade dos processos de gerenciamento. Ou seja, qual é a projeção desejada para a evolução da maturidade em gerenciamento de projetos da organização.

Caso esteja-se utilizando um modelo de maturidade em gerenciamento de projetos, a organização deve compreender bem a realidade descrita por cada nível do modelo. A partir de então, deve verificar qual nível melhor descrever a realidade futura 
na qual a organização deseja estar, com o intuito de caminhar na direção das suas metas estratégicas traçadas. Esta informação é registrada na última linha da Matriz Técnica.

No estudo de campo, os participantes definiram para os três processos de maior contribuição a seguinte capacidade desejada (como mostra parcialmente a Figura 5): P1, nível 3; P2, nível 4; P3, nível 3.

\subsection{Etapa VII - Criar o Plano de Ações}

O objetivo desta etapa é levantar, documentar e priorizar ações de melhoria a partir da priorização das melhorias dos processos de gerenciamento. Nesta etapa, deve-se criar e preencher o segundo conjunto de matrizes do MFD: a House of Actions (HoA). É através desta que as ações de melhoria serão propostas e priorizadas.

A HoA tem como entrada algumas das matrizes preenchidas e calculadas na HoM. A Matriz de Respostas Técnicas da HoM (processos de gerenciamento de projetos) torna-se a primeira matriz da HoA (Matriz de Processos de Gerenciamento de Projetos), enquanto que os dados da Matriz Técnica da HoM (contribuição absoluta, contribuição algébrica, capacidade atual e capacidade desejada) são trazidos para a Matriz de Planejamento da HoA.

A Matriz de Respostas Técnicas e a Matriz de Relacionamentos da HoA não são preenchidas automaticamente. $O$ preenchimento destas segue a mesma idéia das matrizes correspondentes da HoM. A Figura 6 e a Figura 7 mostram a HoA preenchida para os três processos de maior contribuição no estudo de campo.

\begin{tabular}{|c|c|c|c|c|c|c|c|}
\hline $\begin{array}{c}\text { Processos de } \\
\text { Gerenciamento de Projetos }\end{array}$ & \multicolumn{7}{|c|}{ Matriz de Planejamento } \\
\hline 1. Gerenciamento da Integração & Contribuição & $\begin{array}{c}\text { Capacidade } \\
\text { Atual }\end{array}$ & $\begin{array}{l}\text { Capacidade } \\
\text { Desejada }\end{array}$ & $\begin{array}{l}\text { Taxa de } \\
\text { Melhoria }\end{array}$ & $\begin{array}{c}\text { Peso } \\
\text { Absoluto (PA) }\end{array}$ & $\begin{array}{c}\text { PA } \\
\text { Normalizado (PAN) }\end{array}$ & $\begin{array}{c}\text { PAN } \\
\text { Acumulado }\end{array}$ \\
\hline Execução do PGP & 4,26 & 1 & 3 & 3,00 & 12,79 & 0,15 & 0,21 \\
\hline 6. Gerenciamento dos Recursos Humanos & Contribuição & $\begin{array}{c}\text { Capacidade } \\
\text { Atual }\end{array}$ & $\begin{array}{c}\text { Capacidade } \\
\text { Desejada }\end{array}$ & $\begin{array}{l}\text { Taxa de } \\
\text { Melhoria }\end{array}$ & $\begin{array}{c}\text { Peso } \\
\text { Absoluto (PA) }\end{array}$ & $\begin{array}{c}\text { PA } \\
\text { Normalizado (PAN) }\end{array}$ & $\begin{array}{c}\text { PAN } \\
\text { Acumulado }\end{array}$ \\
\hline Aquisição da Equipe & 4,26 & 2 & 4 & 3,00 & 12,79 & 0,15 & 0,71 \\
\hline Desenvolvimento e Gerenciamento do Time do Projeto & 4,26 & 1 & 3 & 3,00 & 12,79 & 0,15 & 0,86 \\
\hline
\end{tabular}

Figura 6 - Matrizes da House of Actions (HoA).

\begin{tabular}{|c|c|c|c|c|}
\hline & & \multicolumn{3}{|c|}{$\begin{array}{c}\text { Matriz de Respostas Técnicas } \\
\text { Ações de Melhoria }\end{array}$} \\
\hline \multicolumn{2}{|c|}{$\begin{array}{c}\text { Processos de } \\
\text { Gerenciamento de Projetos }\end{array}$} & $\begin{array}{l}\text { Relatorios periódicos do } \\
\text { andamento }\end{array}$ & Uso de ferramentas & $\begin{array}{c}\text { Processos bem definidos } \\
\text { e documentados }\end{array}$ \\
\hline \multicolumn{2}{|c|}{ 1. Gerenciamento da Integração } & IMPACTO & IMPACTO & IMPACTO \\
\hline \multicolumn{2}{|c|}{ Execução do PGP } & 3 & 9 & 3 \\
\hline \multicolumn{2}{|c|}{ 6. Gerenciamento dos Recursos Humanos } & IMPACTO & IMPACTO & IMPACTO \\
\hline \multicolumn{2}{|c|}{ Aquisição da Equipe } & 3 & 3 & 9 \\
\hline \multicolumn{2}{|c|}{ Desenvolvimento e Gerenciamento do Time do Projeto } & 3 & 9 & 9 \\
\hline \multirow{2}{*}{ Matriz Técnica } & Contribuição Absoluta: & 1,39 & 3,23 & 3,23 \\
\hline & Contribuiçäo Algébrica: & 1,39 & 3,23 & 3,23 \\
\hline
\end{tabular}

Figura 7 - Matrizes da House of Actions (HoA).

Como se pode observar, durante o estudo de campo, os representantes da organização elegeram três ações de melhoria relacionadas aos três processos de maior contribuição. E dentre estas três ações, as ações A2 (Uso de Ferramentas) e A3 (Processos Bem Definidos e Documentados) tiveram maior contribuição (3,23). 


\subsection{Etapa VIII - Implantar e Acompanhar o Plano de Ações}

O objetivo desta etapa é acompanhar continuamente as ações de melhoria e manter atualizadas as informações contidas nos conjuntos de matrizes do MFD. Ou seja, a oitava e última etapa do MFD representa o final de um ciclo e a preparação para o início de um novo ciclo de melhoria contínua dos processos de gerenciamento de projetos alinhada às estratégias organizacionais.

A partir deste acompanhamento, as matrizes do MFD podem ser atualizadas continuamente, permitindo priorizações cada vez mais precisas e que reflitam a constante evolução da realidade organizacional. Esta etapa não foi vivenciada no estudo de campo, pois estava fora dos objetivos do estudo por limitações de tempo.

\section{Análise Crítica do MFD}

A primeira impressão que os participantes tiveram sobre o MFD foi que "a priorização é muito importante para organizações com muitas metas, pois aparentemente agiliza o processo por funcionar como um guia" (Participante A). Em seguida, os participantes comentaram que concordaram com os resultados apresentados, até porque, as melhorias priorizadas faziam parte do grupo de melhorias que eles informalmente haviam levantado em um momento prévio.

O ponto positivo visualizado pelos participantes no MFD foi a sistematização de um método para identificar as melhorias de processos de gerenciamento e as ações de melhoria mais críticas em função das estratégias traçadas. Como principal ponto negativo, estes levantaram o elevado esforço (tempo consumido) necessário para preencher as matrizes. A partir do depoimento dos participantes, uma análise crítica do MFD foi realizada, que levou à proposição de duas suposições.

Suposição 1 (S1): a sistematização proposta pelo MFD para alinhar estrategicamente a melhoria dos processos de gerenciamento de projetos, formaliza esta tarefa, diminuindo a variabilidade dos resultados obtidos quando comparados com abordagens informais com fins similares.

Suposição 2 (S2): quanto mais metas estratégicas e processos de gerenciamento de projetos forem considerados na análise dos impactos (na HoM e na HoA), mais efetivos serão os resultados do MFD, porém maior será o esforço e o tempo necessário para a construção da HoM e da HoA.

\section{Conclusões e Trabalhos Futuros}

Este artigo apresentou o método Management Function Deployment (MFD) que através da construção, estruturada em uma seqüência de oito etapas, de dois conjuntos de matrizes (House of Management - HoM e House of Actions - HoA) permite alinhar a melhoria dos processos de gerenciamento de projetos às metas estratégicas traçadas por uma organização.

Através de um estudo de campo, realizado em uma empresa de desenvolvimento de software, cada uma das etapas do MFD foi explicada e exemplificada com dados reais. A partir deste estudo de campo, ainda foi possível realizar uma primeira análise crítica do MFD e propor duas suposições a cerca de propriedades peculiares a este método.

Observou-se que o uso do MFD requer um grande esforço e tempo por parte dos representantes da organização envolvida. Porém, acredita-se que este esforço será 
compensado por um programa mais efetivo de melhoria contínua dos processos de gerenciamento de projetos, que auxiliará a organização a caminhar na direção de seus objetivos estratégicos.

Este estudo apresenta conclusões baseadas em um único caso, o que implica que as suposições levantadas neste trabalho devem ser replicadas em outros contextos, semelhantes ou diferentes, a fim de avaliar a sua procedência. Ou seja, a análise baseada em um único caso consiste na principal limitação deste estudo o que sugere que mais experimentos devem ser realizados para (1) verificar em profundidade a viabilidade do uso prático do MFD e (2) analisar a confiabilidade dos resultados obtidos pela aplicação do método. Contudo, apesar desta limitação, o MFD mostrou-se, em primeira instância, um método consistente e coerente e que pode ser aplicado na prática.

Além dos dois trabalhos futuros listados no parágrafo anterior, outros estudos podem ser realizados com o intuito de continuar a linha de pesquisa deste artigo, aperfeiçoando-a e complementando-a: (3) aplicar o MFD em conjunto com outros modelos de maturidade em gerenciamento, (4) executar na prática o ciclo completo de oito etapas do MFD, (5) determinar fatores que influenciam o tempo médio para a execução das etapas do método, (6) criar ferramentas de apoio ao MFD, (7) determinar o impacto de categorias de processos (ex.: processos de gerenciamento de custos) em tipos de metas (ex.: metas financeiras), e (8) realizar estudos experimentais para corroborar, ou refutar, a partir de evidências concretas, as suposições S1 e S2 propostas.

Por fim, é importante destacar a importância do MFD em criar e tornar explícita a preocupação com a melhoria contínua dos processos de gerenciamento de projetos. Uma preocupação nem sempre presente nas organizações. Em particular, uma preocupação não só focada em um ou mais modelos de maturidade, mas direcionada para uma melhoria alinhada às estratégias traçadas pela organização.

\section{Referências}

BECKER, A. L.; PRIKLADNICKI, R.; AUDY, J. L. N. Strategic alignment of software process improvement programs using QFD. In: BiPi '08: Proceedings of the $1^{\text {st }}$ international workshop on Business impact of process improvements. New York, NY, USA: ACM, 2008. p. 9-14.

CARNEVALLI, J. A.; MIGUEL, P. C. Review, analysis and classification of the literature on QFD - types of research, difficulties and benefits. International Journal of Production Economics, v. 114, n. 2, p. 737-754, 2008.

CARVALHO, G.; VASCONCELOS, A.; SILVA, F. Um processo de recomendação de modelos de maturidade em gerenciamento de projetos. In: Proceedings do IV Simpósio Brasileiro de Gerenciamento de Projetos. Ouro Preto, MG, Brasil: PMIMG, 2009. p. 1-10 (Distribuído Digitalmente).

COHEN, L. Quality Function Deployment: How to Make QFD Work for You. Harrisonburg, VA, USA: Addison-Wesley Longman, 1995.

CRAWFORD, J. K. Project Management Maturity Model. 2nd. ed. Pennsylvania, PA, USA: Auerbach Publications, 2006.

DONG-SHENG, S.; FENG-XIA, W. An optimization model of enterprise business processes based on house of quality. In: Proc. International Conference on Management Science and Engineering 2007. Harbin, Heilongjiang, China: ICMSE, 2007. p. 966-971. 
EASTERBROOK, S.; ARANDA, J. Material do curso de Engenharia de Software Experimental. In: 7th International Advanced School on Empirical Software Engineering, Lake Buena Vista, Florida, 2009.

ENGLUND, R. L.; GRAHAM, R. J. From experience: Linking projects to strategy. The Journal of Product Innovation Management, v. 16, p. 52-64, 1999.

HARRISON, F.; LOCK, D. Advanced Project Management: A Structured Approach. 4th. ed. Aldershot, Hampshire, UK: Gower Publishing Ltd, 2004.

KERZNER, H. Using the Project Management Maturity Model: Strategic Planning for Project Management. 2nd. ed. New Jersey, NJ, USA: Wiley, 2005.

LIU, X. et al. QFD application in software process management and improvement based on CMM. In: 3-WoSQ: Proceedings of the third workshop on Software quality. New York, NY, USA: ACM, 2005. p. 1-6.

MILOSEVIC, D. Z.; SRIVANNABOON, S. A theoretical framework for aligning project management with business strategy. Project Management Journal, v. 8, p. 98111, 2006.

OGC. PRINCE2 Maturity Model. UK: Office of Government Commerce, 2006.

OGC. Portfolio, Programme and Project Management Maturity Model. UK: Office of Government Commerce, 2008.

PINTO, A. Estudo de Benchmarking em Gerenciamento de Projetos - Relatório Técnico dos Chapters Brasileiros do Project Management Institute. Brasil, 2008.

PMI. Organizational Project Management Maturity Model (OPM3) - Knowledge Foundation (2nd Edition). 2nd. ed. Pennsylvania, PA, USA: Project Management Institute, 2008.

PRADO, D. Maturidade em Gerenciamento de Projetos. Minas Gerais, MG, Brasil: INDG Tecnologia e Serviços, 2008.

SRIVANNABOON, S. Linking project management with business strategy. In: PMI Global Congress (North America). Washington, WA, USA: Project Management Institute, 2006.

YUMING, Z.; QUAN, P.; GUO, P. Research on the Application of Project Portfolio Management (PPM), Program Management (PM) and Project Management in Enterprise Strategic Management. In: Proc. International Conference on Wireless Communications, Networking and Mobile Computing WiCom 2007. Shanghai, P.R. China: WiCom, 2007. p. 5266-5269.

ZHAO, Y. et al. The application of extend QFD in BPR. In: Proc. IEEE International Conference on Systems, Man and Cybernetics. Washington, DC, USA: SMC, 2003. v. 5 , p. $4742-4747$. 